6 Burdett S, Pignon JP, Tierney J, et al. Adjuvant chemotherapy for resected early-stage non-small cell lung cancer. Cochrane Database Syst Rev 2015; 3: CD011430.

7 Cai XW, Xu LY, Wang L, et al. Comparative survival in patients with postresection recurrent versus newly diagnosed non-small-cell lung cancer treated with radiotherapy. Int J Radiat Oncol Biol Phys 2010; 76: 1100-1105.

8 Law MR, Henk JM, Lennox SC, et al. Value of radiotherapy for tumour on the bronchial stump after resection for bronchial carcinoma. Thorax 1982; 37: 496-499.

9 Kono K, Murakami M, Sasaki R, et al. [Radiation therapy for non-small cell lung cancer with postoperative intrathoracic recurrence]. Nihon Igaku Hoshasen Gakkai Zasshi 1998; 58: 18-24.

10 Kelsey CR, Clough RW, Marks LB. Local recurrence following initial resection of NSCLC: salvage is possible with radiation therapy. Cancer J 2006; 12: 283-288.

\title{
Radiation therapy for post-operative recurrence: yes, but only for limited indications
}

\section{From the authors:}

We read the correspondence of Cihoric and colleagues related to our editorial with great interest. Radiation therapy, either alone or in combination with chemotherapy, is a widely accepted treatment modality for post-operative lung cancer relapse. The focus of our editorial was to increase the awareness of possible repeat intervention in this quite limited patient group with generally poor prognosis [1]. Although the overall message of this correspondence is not debatable, the statement that "the available evidence shows that patients with post-resection recurrent nonsmall cell lung cancer (NSCLC) can achieve excellent results when treated with radiation therapy alone, or with radio-chemotherapy" is somewhat misleading as we have several concerns related to the evidence supporting the above statement.

The analysis cited under reference 5 in the correspondence included patients treated at the radiation therapy department in the period 1982-1993 [2]. In this series, both preoperative and post-recurrence work-up was unclear as computed tomography scans only became mandatory after 1988 . Selection criteria for initial surgery are unknown, as well as criteria to reject patients with relapse from repeat surgery. With such an inconsistent or unclear preoperative work-up, well before the era of positron emission tomography and mediastinoscopy, it does not seem appropriate to compare survival of patients with isolated bronchial stump recurrences with survival of patients with surgery alone (or combined with other therapeutic modalities) in newly diagnosed T2N0 NSCLC.

Furthermore, comparison of survival of patients with post-surgery recurrence versus newly diagnosed NSCLC patients treated with radiation therapy or combined radio-/chemotherapy is not very helpful in optimising a therapeutic approach for cases of post-operative relapse [3].

We agree that combined radio-/chemotherapy is a promising option for patients with a post-surgical recurrence, as in the single-centre study of TAKENAKA et al. [4], but studies comparing repeat surgery with radio-/chemotherapy or with radiation therapy alone are missing. Similarly, the results of the systematic review cited under the reference 3 in the correspondence from Cihoric and colleagues, with median survival times of $\sim 28.5$ months and a 5-year survival in excess of $30 \%$, emerged from considerations of treatment modalities with radiation therapy alone [5].

In addition, there are some general concerns when comparing surgery with the newly introduced stereotactic radiation therapy: specific pathological diagnosis is not obtained in every case; lymph node evaluation is less rigorously performed; and evaluation of treatment is quite challenging after stereotactic radiation therapy due to the inflammatory response and fibrosis that invariably occur [6, 7]. After complete surgical resection this is more straightforward as there is no remaining disease. For this reason direct comparison between surgery and radiation therapy is not possible [7].

In conclusion, radiation treatment remains an alternative treatment option for post-surgical lung cancer relapse, especially for those patients that are functionally inoperable or technically unresectable. Further evidence is certainly needed to develop more general recommendations for treatment of recurrent disease. 
$@$ ERSpublications

Radiation therapy remains an alternative treatment option for post-surgical lung cancer relapse http://ow.ly/GaM43006HDs

Dragan Subotic ${ }^{1}$, Paul Van Schil ${ }^{2}$ and Bogdan Grigoriu ${ }^{3}$

${ }^{1}$ Clinic of Thoracic Surgery, Clinical Center of Serbia, University of Belgrade Faculty of Medicine, Belgrade, Serbia.

${ }^{2}$ Dept of Thoracic and Vascular Surgery, University Hospital, Antwerp, Belgium. ${ }^{3}$ Dept of Pulmonology, University of

Medicine and Pharmacy, Iasi, Romania.

Correspondence: Dragan Subotic, Clinic of Thoracic Surgery, Clinical Center of Serbia, University of Belgrade Faculty of Medicine, Koste Todorovica 26, 11000 Belgrade, Serbia. E-mail: profsubotic@gmail.com

Received: April 292016 | Accepted: May 042016

Conflict of interest: None declared.

\section{References}

1 Subotic D, Van Schil P, Grigoriu B. Optimising treatment for post-operative lung cancer recurrence. Eur Respir J 2016; 47: 374-378.

2 Jeremic B, Shibamoto Y, Milicic B, et al. External beam radiation therapy alone for loco-regional recurrence of non-small-cell lung cancer after complete resection. Lung Cancer 1999; 23: 135-142.

3 Cai XW, Xu LY, Wang L, et al. Comparative survival in patients with postresection recurrent versus newly diagnosed nonsmall-cell lung cancer treated with radiotherapy. Int J Radiat Oncol Biol Phys 2010; 76: 1100-1105.

4 Takenaka T, Takenoyama M, Toyozawa R, et al. Concurrent chemoradiotherapy for patients with postoperative recurrence of surgically resected non-small-cell lung cancer. Clin Lung Cancer 2015; 16: 51-56.

5 Jeremic B, Bamberg M. External beam radiation therapy for bronchial stump recurrence of non-small-cell lung cancer after complete resection. Radiother Oncol 2002; 64: 251-257.

6 Van Schil PE, Van Meerbeeck J. Surgery or radiotherapy for early-stage lung cancer - a potential comparison bias. Lancet Oncol 2013; 14: e390.

7 Van Schil PE. Results of surgery for lung cancer compared with radiotherapy: do we speak the same language. J Thorac Oncol 2013; 8: 129-130.

Q

CrossMark

\section{Readmission in COPD patients: should we consider it a marker of quality of care or a marker of a more severe disease with a worse prognosis?}

\section{To the Editor:}

In the January edition of the European Respiratory Journal, we read with interest the paper by HARTL et al. [1], which evaluated, from the European COPD Audit, patients with acute exacerbation of chronic obstructive pulmonary disease (AECOPD) and the mortality risk during hospitalisation and in the post-discharge period of 90 days. Moreover, the authors evaluate the risk of readmission in a period of 90 days from discharge.

The readmission to hospital that occurs with COPD patients is a many-sided phenomenon, where the complexity of patients and the difficulties with the healthcare systems are often considered as causes [2]. The reasons of readmission may be respiratory and non-respiratory related [2]. Starting from the concept that in COPD patients, like for other chronic diseases with high costs related to discharge (congestive heart failure, acute myocardial infarction and pneumonia), the early readmissions (30-days from discharge) should be considered a marker of quality of care that patients receiving during hospitalisation [3], in USA by the Centres for Medicare and Medicaid Services financial penalties were applied for hospitals with high rates of readmissions [4]. Several interventions have been proposed [5], even if a single disease-specific approach is not recommended [2,5]; obviously, data about prediction of readmission might be extremely useful $[6,7]$.

Although the study by HARTL et al. [1] focuses on a period of 90 days from discharge and the causes of readmission are not exclusively respiratory related, our recently published long-term observational study [8] 